\title{
Use of the home cage as an anesthesia induction chamber
}

Dr. Ella Mann had an active research program for nearly 40 years. As retirement age was nearing, she volunteered to serve on the Great Eastern University IACUC, an undertaking that interested her but that she had not had time to pursue before. After a training period, the first protocol she received for review involved a surgical procedure with mice. The description of the isoflurane anesthesia induction included putting the mouse into a special induction chamber and gradually filling the chamber with isoflurane.
This seemed strange to Mann, whose research had required euthanizing mice but never anesthetizing them for surgery. The Great Eastern policy for carbon dioxide or isoflurane euthanasia of mice included keeping them in their home cages to reduce possible distress to the animals. Mann reasoned that if it was potentially distressful to euthanize a mouse in a chamber other than its home cage, then it would also be potentially distressful to anesthetize a mouse in a chamber other than its home cage. Wondering why the IACUC did not require the use of the home cage as an induction chamber, she asked the school's attending veterinarian. When the veterinarian wasn't able to provide a cogent response, Mann asked the IACUC chair, and when he suggested that she ask the veterinarian, Mann knew she had touched upon a sensitive subject for the IACUC.

Do you think Mann has a valid concern about the method of anesthesia induction? How should the IACUC approach the question raised by Mann?

\section{RESPONSE}

\section{Chamber is preferred}

Jennifer N. Davis, DVM, DACLAM, Adam Caro, DVM, DACLAM, Stella Spears \& Julie Sharp, DVM, CPIA, DACLAM

This scenario exemplifies how IACUC members apply great thought, judgment and responsibility in weighing the use of animals in research. Individual IACUC members might not always agree on specific details surrounding protocols and animal use procedures, and dissenting views should be acknowledged and discussed by the committee. In this situation, Mann has a valid concern regarding the welfare of the animals. The IACUC should weigh the advantages and disadvantages of anesthetizing an animal in its home cage versus an induction chamber. A variety of factors should be considered by the IACUC when determining the best approach for anesthesia: the number of animals to be anesthetized, the outcome of the surgical procedure (non-survival or survival), minimization of pain and stress to an animal upon recovery and potential for injury during induction.
The 2013 AVMA Guidelines for Euthanasia of Animals acknowledge that whenever possible, inhaled agents should be administered under conditions where animals are most comfortable $^{1}$ (e.g., for rodents, in the home cage), a recognized best practice to produce the least aversive experience for the animals undergoing euthanasia. A cage may contain a single animal or a group of socially housed animals. Most often, an individual animal from a socially housed group is anesthetized. In these cases, it is more reasonable to remove that animal from the home cage to an induction chamber for the procedure. In this way, only one animal is relocated to a new environment, minimizing stress to the remaining animals.

If the animals were being anesthetized to undergo a survival surgery, then use of an induction chamber could help to minimize stress during recovery. During anesthetic induction, a mouse might release stress hormones into the environment. If this environment is the home cage, then the animal would be exposed to those hormones upon return to its home cage after surgery and might experience additional stress.

When inducing anesthesia, the safety of the environment should also be considered. Contents of the home cage, such as bedding and enrichment devices, can inhibit visualization of the mouse, hindering observers' ability to evaluate the anesthetic plane, and can also obstruct the nares, cause corneal injury or become lodged in the animal's mouth if the transition to unconsciousness is not quick and smooth. In contrast, an induction chamber permits clear visualization and continuous monitoring of the animal.

We feel that removing an animal from its home cage and inducing anesthesia within a specialized chamber is preferred. This practice minimizes stress to the animal and its cage-mates, is a safe way to anesthetize individual animals and allows animals to recover in a familiar home cage. The IACUC must weigh protocols carefully and consider the potential pain and stress experienced both by an individual animal and by other animals in the same cage, with the goal of minimizing pain and stress to all animals.

1. American Veterinary Medical Association. AVMA Guidelines for the Euthanasia of Animals: 2013 Edition (American Veterinary Medical Association, Schaumburg, IL, 2013).

Davis is Assistant Director for Compliance, Caro is Protocol Specialist Veterinarian, Spears is DVM candidate and veterinary extern, and Sharp is Interim Director in the Office of Animal Welfare, University of Pennsylvania, Philadelphia, PA. 